\title{
Transnasal penetrating intracranial injury with a chopstick
}

\author{
SK Chan *, KY Pang, CK Wong
}

\section{A B S T R A C T}

We report the first case of a transnasal penetrating intracranial injury in Hong Kong by a chopstick. A 49-year-old man attempted suicide by inserting a wooden chopstick into his left nose and then pulled it out. The chopstick caused a transnasal penetrating brain injury, confirmed by contrast magnetic resonance imaging of the brain. He was managed conservatively. Later he developed meningitis without a brain abscess and was prescribed antibiotics for 6 weeks. He enjoyed a good neurological recovery. This case illustrates that clinician should have a high index of suspicion for penetrating intracranial injury due to a nasally inserted foreign body, even though it had already been removed. In such cases moreover, brain magnetic resonance imaging is the imaging modality of choice, as it can delineate the path of penetration far better than plain computed tomography.

\section{Hong Kong Med J 2014;20:67-9}

DOI: 10.12809/hkmj134028

SK Chan *, MB, BS

KY Pang, FRCS, FHKAM (Surgery)

CK Wong, FRCS, FHKAM (Surgery)

Department of Neurosurgery, Pamela Youde Nethersole Eastern Hospital, Chai Wan, Hong Kong

* Corresponding author: skc2009@hotmail.com

\section{Case report}

A 49-year-old Chinese man, who was a psychiatric in-patient, self-inserted a wooden chopstick into his

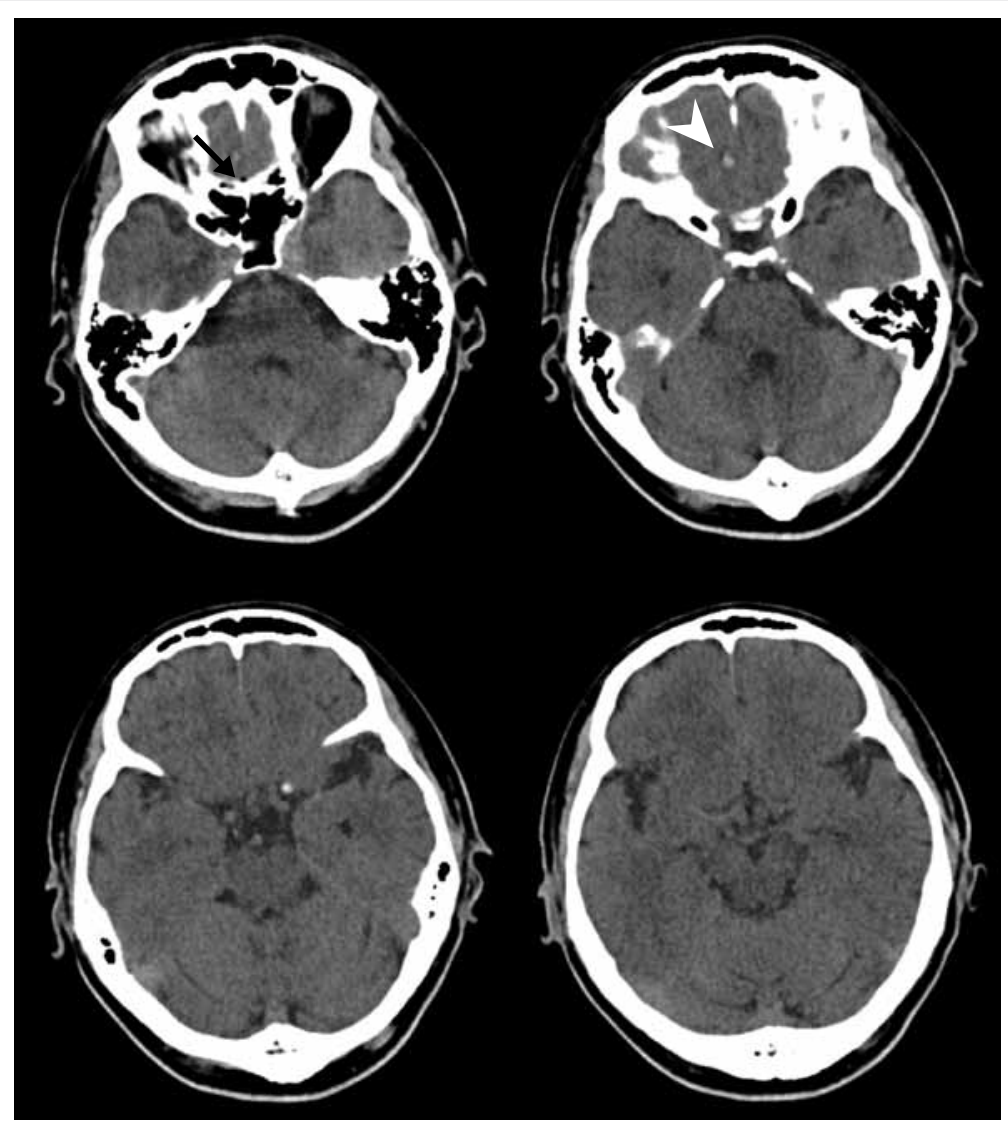

FIG I. Plain computed tomographic scan of brain (axial cut) showing pneumocephalus (black arrow) and haemorrhage over right gyrus rectus (white arrowhead) left nose and then pulled it out in November 2012. He was subsequently assessed by an ear, nose and throat surgeon. No nasal foreign body was seen and there was no epistaxis. Brain computed tomography (CT) 6 hours after the injury showed a trace amount of haemorrhage over the right gyrus rectus and a small amount of pneumocephalus over the right anterior fossa (Fig 1), and hence the neurosurgical unit was consulted. Six hours after the incident his vital signs were stable and he was afebrile; his Glasgow Coma Scale score was E4M6V4. His speech appeared confused, as if in a premorbid state. There was no neurological deficit, and no cerebrospinal fluid (CSF) rhinorrhoea upon stress testing. Contrast magnetic resonance imaging (MRI) of the brain was performed on the next day, which showed a long haemorrhagic tract extending from right paramidline anterior skull base, coursing postero-superiorly across medial right frontal lobe, closely adjacent to the right frontal horn, and ending at the vertex region (Fig 2), and a trace of intraventricular haemorrhage. On the same day he developed fever. Lumbar puncture yielded turbid CSF, with the presence of Gram-positive cocci, as well as low CSF glucose and high CSF protein concentrations. Conservative management was adopted. On an empirical basis, intravenous ceftriaxone, vancomycin, and metronidazole were prescribed. Prophylactic anticonvulsant therapy (phenytoin) was also given. The CSF culture grew Staphylococcus aureus and Citrobacter koseri. The antibiotic regimen was switched to intravenous ceftriaxone and oral metronidazole. The patient's recovery was excellent, as reflected by normalisation of body temperature and inflammatory markers. On 


\section{筷子穿過鼻孔導致虜內損傷 \\ 陳錫鈞、彭佳源、黃志強}

本文報告首宗在香港發生以筷子穿透鼻孔致顱內損傷的案例。一名49 歲男子自殺時把一支木筷子插入他左邊的鼻孔, 然後再把筷子拔出 來。其後腦部磁力共振證實顱內損傷, 遂施以保守治療。後來病人感 染腦膜炎, 經為期六週的抗生素治療後, 康復理想。本病例説明醫生 必須對插入鼻孔的異物（即使已被移走）要有高度警覺性, 因為這些 異物可能導致顱內損傷。由於臚內損傷在腦部磁力共振下比電腦斷層 掃描明顯得多, 腦磁力共振是臚內損傷的首選影像檢查。
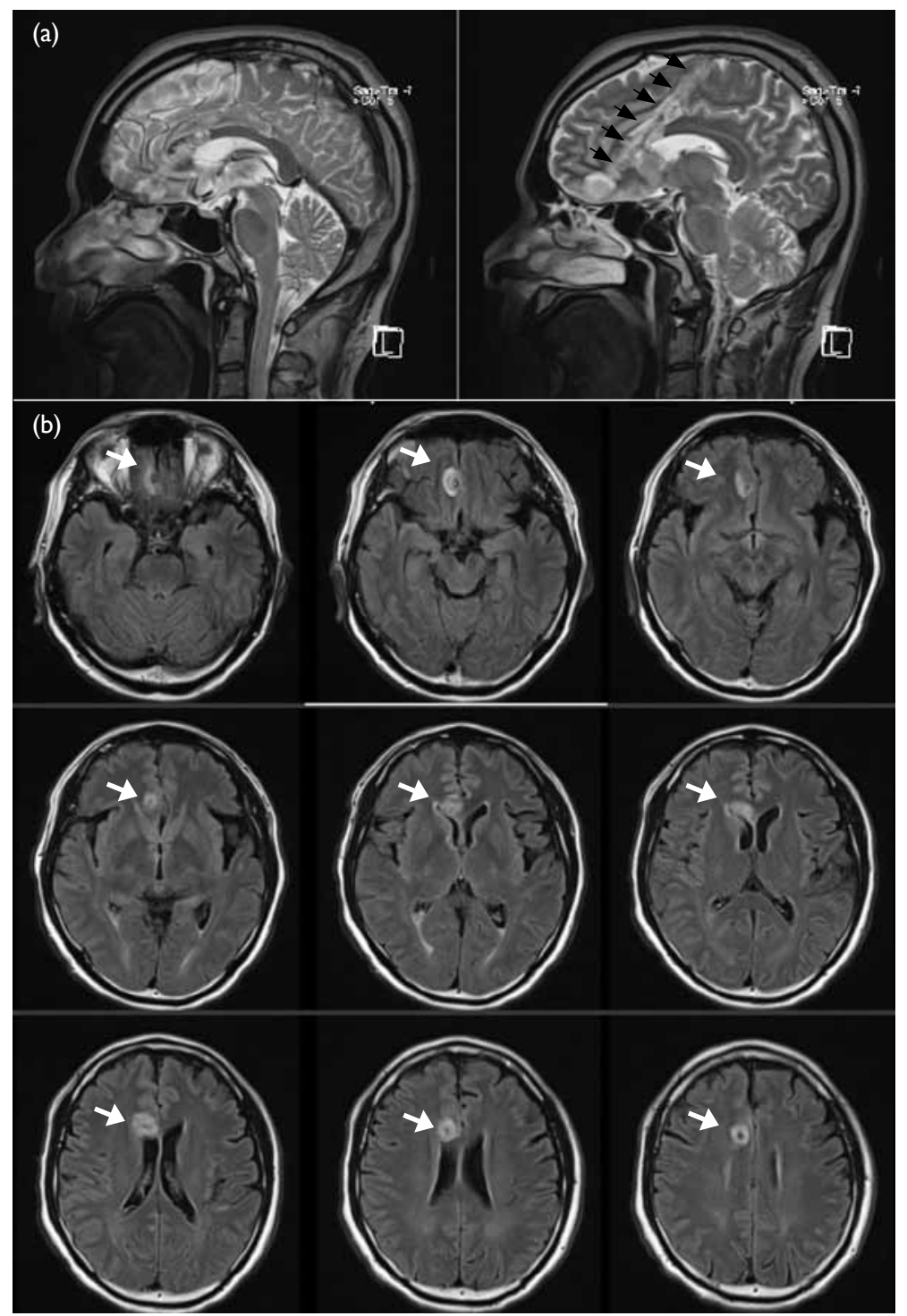

FIG 2. (a) T2-weighted magnetic resonance imaging (MRI) scan of brain (sagittal cut) showing transected track (black arrows). (b) T2-weighted MRI scan of brain in fluid-attenuated inversion recovery sequence (axial cut) showing transected track (white arrows) day 14, contrast CT brain showed no abscess. He was well after 6 weeks of antibiotic treatment.

\section{Discussion}

A literature search of the MEDLINE database (using the key words "transnasal" and "penetrating intracranial injury", or the other similar keywords) was conducted. Approximately 10 case reports were identified, indicating this was an exceedingly rare condition. We report the first case of a transnasal penetrating intracranial injury in Hong Kong by means of a chopstick. The majority of patients with this mechanism of injury were due to accidental falls on objects that enter the nostril, ${ }^{1}$ while few were due to attempted suicide. This mode of penetrating intracranial injury can result in severe complications, including CSF rhinorrhoea and infection (meningitis and/or brain abscess). ${ }^{2}$ The source of the bacteria could have been from the chopstick or the normal flora in the nasal cavity/paranasal sinuses. Such injury can also result in blindness or ophthalmoplegia, ${ }^{3}$ if the orbital cavity is involved. The most dreadful complication is severe haemorrhage due to internal carotid artery injury, which may lead to immediate death. ${ }^{1}$ Although foreign body in the nose is a common presentation, clinicians should always have a high index of suspicion for possible transnasal penetrating intracranial injury. Such vigilance is especially necessary in patients who cannot volunteer a clear history or complaint (eg children or psychiatric patients), are febrile, have a neurological deficit, or have continuous epistaxis.

All patients with penetrating brain injury should have adequate neuroradiological examinations. $^{4}$ Plain CT brain is the initial investigation of choice to explore for the presence of any retained radio-opaque material. It can also reveal presence of an intracranial haematoma or air, or focal heterogeneous hypodensity suggestive of brain abscess. Contrast MRI of the brain with cerebral angiography is the best method of evaluating penetrating brain injuries. Besides the previously mentioned abnormal findings, it can also delineate the track transected by the foreign body, even after it has been removed, as in this case. Had contrast MRI brain not been arranged for this patient, penetrating brain injury would probably have been missed, given the seemingly unalarming brain CT (Fig 1). Brain abscess or vascular damage, if any, can also be appreciated using brain MRI.

Management depends on patient's condition and whether a complication has developed. Neuroendoscopy is a useful operative tool, which can be employed to remove foreign body or repair the dural defect causing CSF rhinorrhoea, ${ }^{5}$ whenever conservative management fails. Craniotomy may be warranted if there is a sizable abscess or cerebral haemorrhage. Since the patient did not have these 
complications, he was managed conservatively. Initially, he received empirical intravenous broadspectrum antibiotics, as for the treatment of a bacterial cerebral abscess. The CSF should be obtained and cultured if the patient has a fever. Targeted antibiotics can then be used depending on the sensitivity results. The patient was also instructed to lie flat to prevent CSF leakage. Shortterm prophylactic anticonvulsant should also be prescribed.

\section{Conclusion}

Herein we report the first patient with a transnasal penetrating intracranial injury in Hong Kong resulting from a chopstick. Such injuries confer significant mortality or morbidity, for which clinicians should always have a high index of suspicion. The neuroimaging of choice involves contrast MRI brain with cerebral angiography.
Operative management can be considered for those patients who have a foreign body in situ, a sizable abscess or haemorrhage, or CSF rhinorrhoea.

\section{References}

1. Ihama Y, Nagai T, Ninomiya K, Fukasawa M, Fuke C, Miyazaki T. A transnasal intracranial stab wound by a plastic-covered umbrella tip. Forensic Sci Int 2012;214:e9e11.

2. Hiraishi T, Tomikawa M, Kobayashi T, Kawaquchi $\mathrm{T}$. Delayed brain abscess after penetrating transorbital injury [in Japanese]. No Shinkei Geka 2007;35:481-6.

3. Liu SY, Cheng WY, Lee HT, Shen CC. Endonasal transsphenoidal endoscopy-assisted removal of a shotgun pellet in the sphenoid sinus: a case report. Surg Neurol 2008;70 Suppl 1:S1:56-9.

4. Sharif S, Roberts G, Phillips J. Transnasal penetrating brain injury with a ball-pen. Br J Neurosurg 2000;14:159-60.

5. Lee DH, Seo BR, Lim SC. Endoscopic treatment of transnasal intracranial penetrating foreign body. J Craniofac Surg 2011;22:1800-1. 